\title{
Cutaneous Mycobacterium chelonae in a Patient with Sjogren's Syndrome
}

\author{
Lu Guo' \\ Sha Lv' \\ Jing Zeng' \\ Jiayu Yang ${ }^{2}$ \\ Baihui Shan' \\ Fuqiu Li' \\ 'Department of Dermatology, The \\ Second Hospital of Jilin University, \\ Changchun, Jilin, People's Republic of \\ China; ${ }^{2}$ Department of Obstetrics and \\ Gynecology, China-Japan Union Hospital \\ of Jilin University, Changchun, Jilin, \\ People's Republic of China
}

\begin{abstract}
A case of cutaneous Mycobacterium chelonae infection in a patient with Sjogren's syndrome (SS) was misdiagnosed as sporotrichosis. A 56-year-old female patient was admitted to another hospital. Based on results of the histopathological examination and secretion culture obtained at the other hospital, the patient was diagnosed with sporotrichosis and received antifungal therapy. After treatment failure, the patient was admitted to our hospital, and a histopathological examination and secretion culture were performed again. The secretion culture revealed the presence of Mycobacterium chelonae. The antinuclear antibody test suggested SS, and the patient was treated with antibiotics and corticosteroids.
\end{abstract}

Keywords: Mycobacterium chelonae, cutaneous infection

\section{Introduction}

Mycobacterium chelonae is a nontuberculous mycobacterium (NTM) that is classified as a rapidly growing mycobacterium according to the Runyon classification. Atypical disseminated and systemic mycobacterial infections mainly occur in patients with low immune function, autoimmune diseases, and long-term cortisoluse. $^{1,2}$ To the best of our knowledge, an atypical mycobacterial infection in a patient with SS has not been previously reported. Here, we describe a rare, previously unreported case of cutaneous Mycobacterium chelonae infection in a patient with SS.

\section{Case Presentation}

A 56-year-old postmenopausal female patient was admitted to another hospital for "multiple dark red painful nodules on the limbs for 2 months". Two months prior, the patient developed several dark red papules, nodules and granulomatous changes in the limbs with no obvious cause. A pathological examination indicated mixed cellular granuloma in the whole dermis, and secretion culture yielded white, smooth, yeast-like colonies. Based on the results of the histopathological examination and secretion culture, the patient was diagnosed with sporotrichosis, and itraconazole $(200 \mathrm{mg} /$ time, BID) was orally administered.

The symptoms did not resolve, and the patient was admitted to our hospital one month later. The number of nodules on her limbs had increased, and some of the nodules appeared to be due to hyperplasia. Ulcerations with purulent secretions were observed, and the patient reported significant pain and fatigue. Specialist examination findings were as follows: swelling and purulent exudation from the wrist (Figure 1A) to the left forearm (Figure 1B); beaded proliferative nodules from the dorsum of the right
Correspondence: Fuqiu Li

Tel +8613039123758

Fax +86-43I-8II36888

Email lifuqiu1234@126.com 


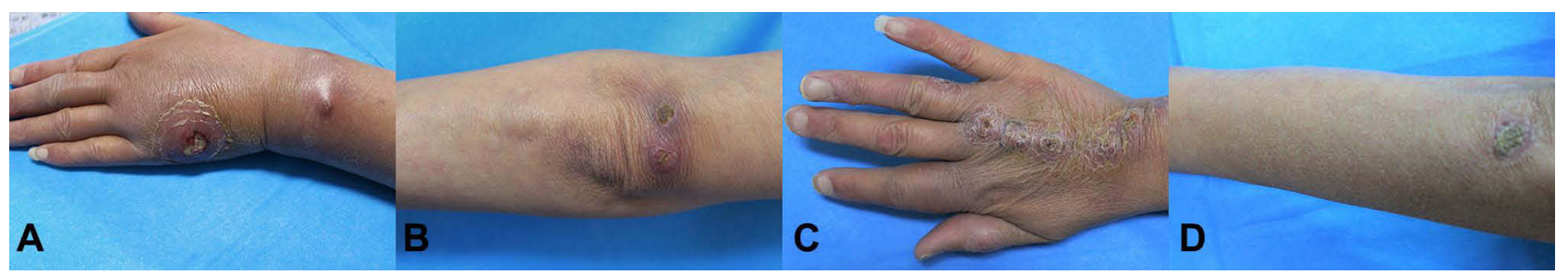

Figure I Swelling and purulent exudation from the wrist $(\mathbf{A})$ to the left forearm (B); beaded proliferative nodules from the dorsum of the right hand (C) to the right forearm (D).

hand (Figure 1C) to the right forearm (Figure 1D); and scattered nodules on both knees (Figure 2A) and the left lower limb (Figure 2B). The patient's medical history was as follows: diagnosed with right lateral malleolar tuberculosis with synovitis and anemia 8 years previously; diagnosed with hypothyroidism. She reported stiff fingers, pale and blue fingers when cold for 8 years, dry mouth, dry eyes and fatigue for 2 years. The patient denied any history of trauma or mosquito bites. Upon admission to our hospital, histopathological examination and secretion culture were performed again. The histopathological results showed superficial and deep granulomatous dermatitis with suppuration, multinucleated giant cells (Figure 3A); and negative periodic acid Schiff (PAS) staining (Figure 3B). Acid-fast bacilli were seen on skin biopsy stained with Ziehl-Neelsen stain (Figure 3C). White, smooth colonies were observed in potato dextrose agar (PDA) medium after 1 week (Figure 4). Acid-fast staining of the bacterial suspension demonstrated acid-fast bacilli (Figure 5). The auxiliary examination findings were as follows: antinuclear antibody (ANA) detection: nucleoprotein U1RNP antibody $(+++)$, Sjogren's syndrome A (SSA) antibody $(+++)$, and 52-kDa protein antibody (++ + ); ANA fluorescence results: nuclear particle type (1:1000 positive) and cytoplasmic particle type (1:3200 positive). The diagnosis was atypical cutaneous mycobacterial infection and SS. The following drugs were administered: $5 \mathrm{mg}$ betamethasone (intramuscular injection), $450 \mathrm{mg}$ rifampicin once/d (oral), and $500 \mathrm{mg}$ clarithromycin once/d (oral). The colonies on the PDA medium were sent to Shenggong Bioengineering (Shanghai) Co., Ltd. Genomic DNA was extracted, amplified and sequenced with $16 \mathrm{~S}$ rRNA primers (upstream 5'-CagagttTGATCCTGGCT-3', downstream 5'AggaggTGATCCAGCCGCA-3'). A Basic Local Alignment Search Tool (BLAST) comparison was performed in GenBank, and 100\% homology with Mycobacterium chelonae was revealed. The patient was diagnosed with cutaneous Mycobacterium chelonae infection and SS. Due to economic reasons, the patient did not attend a follow-up examination at our hospital. The patient was informed by telephone to change the treatment to $500 \mathrm{mg}$ clarithromycin once/d, and 500mg ciprofloxacin twice/d. Additional telephone follow-ups are in progress.

\section{Discussion}

Mycobacterium chelonae is most commonly found in soil and water and on poorly sterilized medical equipment and

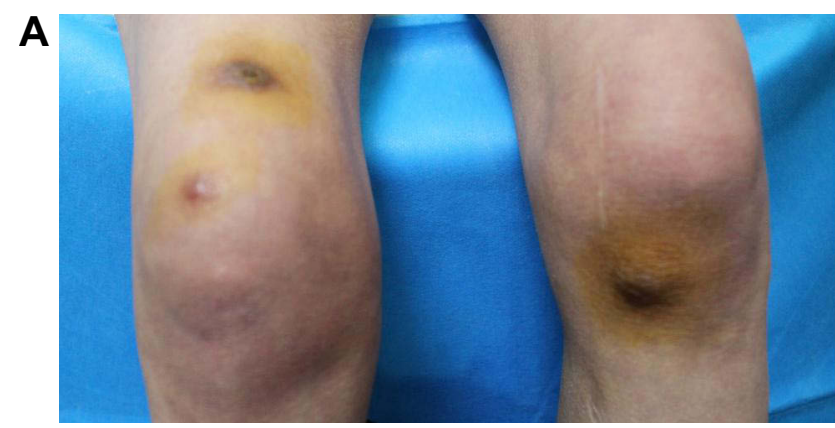

B

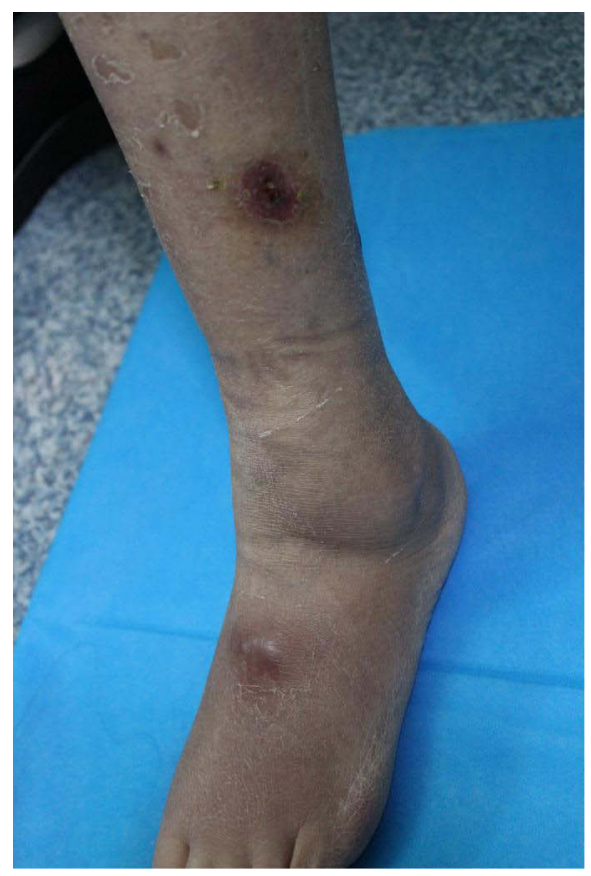

Figure 2 Scattered nodules on both knees (A) and the left lower limb (B). 


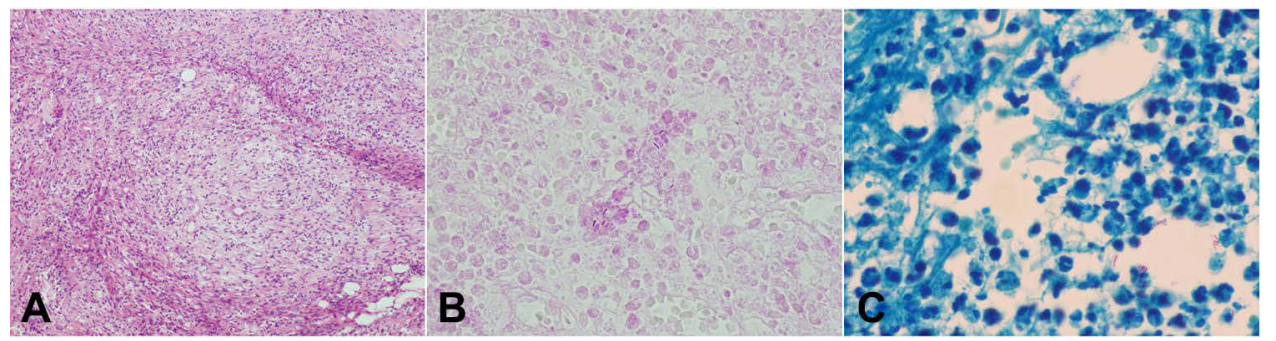

Figure 3 The histopathological results showed superficial and deep granulomatous dermatitis with suppuration, multinucleated giant cells (A); negative periodic acid Schiff (PAS) staining (B); acid-fast bacilli were seen on skin biopsy stained with Ziehl-Neelsen stain (C).

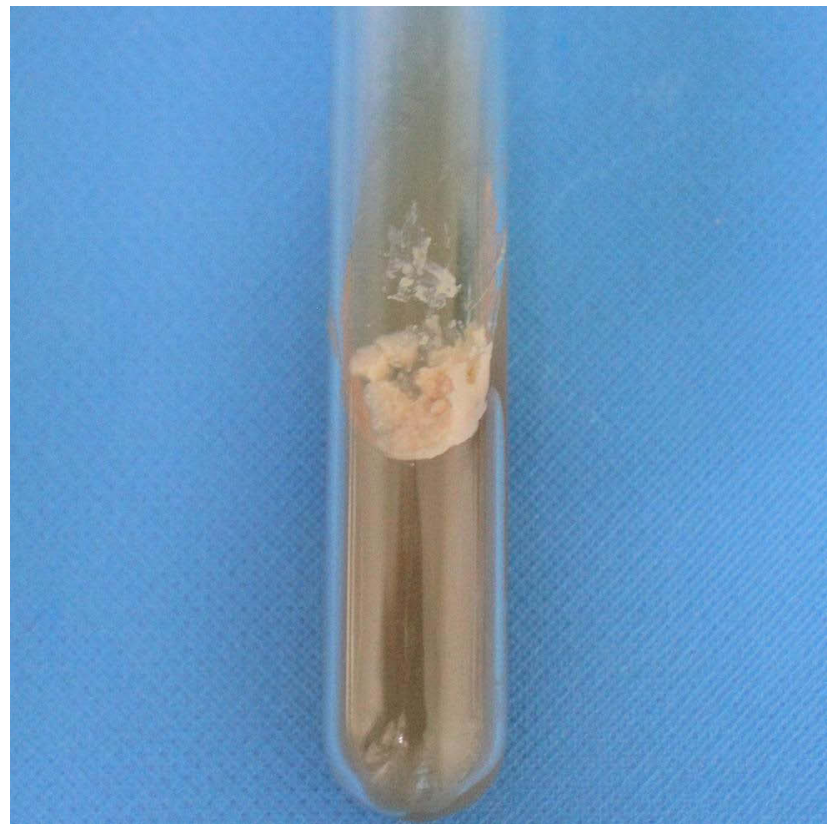

Figure 4 White, smooth colonies was observed in potato dextrose agar (PDA) medium after I week.



Figure 5 Acid-fast staining of the bacterial suspension demonstrated acid-fast bacilli. can cause infection in different organs, such as the skin and soft tissue, lymphatic system, and lungs, it can also cause disseminated infection and some rare types of infections. ${ }^{3}$ The most common infections are skin and soft tissue infections. Because this bacterium has a tendency to grow at low temperatures, skin lesions often appear on the extremities and can manifest as erythema, papules, nodules, abscesses, nodular lymphangitis, and vasculitis. ${ }^{4}$

The key to diagnosing Mycobacterium chelonae infection is etiological identification using, morphological, high-performance liquid chromatography and molecular biology techniques. ${ }^{5}$ The disadvantage of morphological identification is that culture requires a long time, and the positivity rate is affected by sampling techniques, the culture medium and early drug use. Molecular identification can be used for early and rapid diagnosis. When atypical mycobacterial infection is clinically suspected, early molecular biological identification can be used to confirm the diagnosis. Atypical mycobacterial infection is rare, and the clinical manifestations are very similar to those of sporotrichosis, especially lymphangitic sporotrichosis. Skin pathological features include nonspecific mixed, cellular, and inflammatory granulomas in the dermis. ${ }^{6}$ Culture requires a long time, and the colonies can be morphologically similar to Sporotrodix colonies. Thus, the disease is easy to misdiagnose. For example, at this patient's first visit, she were diagnosed as sporotrichosis in the other hospital. The patient did not respond to antifungal treatment and was admitted to our hospital. We questioned the initial diagnosis, and repeated pathological acid-fast staining suggested a mycobacterial infection, which was confirmed by molecular biological methods as a Mycobacterium chelonae infection. There is no report of atypical mycobacterium growing on PDA medium. 
Mycobacterium chelonae is more resistant to antibiotics than other rapidly growing mycobacteria. It is highly sensitive to clarithromycin; moderately sensitive to ciprofloxacin, ofloxacin and amikacin; and naturally resistant to rifampicin and isoniazid. The empirical treatment was combined clarithromycin and amikacin for at least 4 months and not exceeding 12 months with debridement and drainage when necessary.

Atypical disseminated and systemic mycobacterial infections occur mainly in patients with low immune function, autoimmune diseases, and long-term cortisol-use. ${ }^{1,2}$ The patient was diagnosed as Sjogren's syndrome according to her medical history and molecular examination results. But she has no history of cortisol-use. SS is a systemic autoimmune disease characterized by dry eyes and dry mouth due to inflammation and lesions in the lacrimal and salivary glands. It also affects the joints, skin, lungs, gastrointestinal tract, nervous system, and kidneys. ${ }^{7}$ Corticosteroids and hydroxychloroquine can improve the symptoms of SS. ${ }^{8}$

Atypical mycobacterial infection with SS has not been previously reported. It has been reported that exposure to mycobacteria may be related to the pathogenesis of SS; thus, it is hypothesized that the bacilli Calmette-Guerin (BCG) vaccine and antimycobacterial drugs may contribute to the prevention and treatment of SS. ${ }^{9}$ According to Chao et al, a correlation exists between NTM infection and SS, and this correlation is very high in postmenopausal and nonbronchiectasis subjects. ${ }^{10}$ The mechanism of this correlation may be related to type I interferon and sex hormones. In SS patients with enlarged salivary glands, positive anti-Ro /SSA titers and decreased lymphocytes, increased type I interferon (IFN), and upregulation of type I IFN-induced genes were noted. ${ }^{11}$ Rayamajhi et al found antagonistic crosstalk between type I and type II IFN; that is, IFN $\alpha \beta$ can inhibit the host's response to IFN $\gamma$ and increase susceptibility to mycobacteria. ${ }^{12}$ Low levels of serum sex hormones, namely dehydroepiandrosterone (DHEA), have been found in women with pulmonary mycobacterial complex infection, and the lack of estrogen and DHEA will lead to increased apoptosis of exocrine secretory cells. ${ }^{10}$ These mechanisms can explain the high correlation between NTM infection and SS in postmenopausal women. ${ }^{10}$ Correspondingly, this patient is a postmenopausal woman who got infected with Mycobacterium chelonae and Sjogren's syndrome.

\section{Conclusion}

A prompt molecular diagnosis of atypical mycobacterial infection is required to initiate appropriate treatment.
Identification of the causative agent is important because it can guide further medical treatment. In addition, this case suggests that patients with disseminated or systemic atypical mycobacterial infection should be screened for autoimmune diseases as well as a history of immune deficiency and hormone use. According to Chao et al, there is a high correlation between NTM infection and SS in postmenopausal women, and its mechanism may be related to type I IFN and sex hormones. The specific immune mechanism needs further study.

\section{Abbreviations}

PAS, periodic acid Schiff; PDA, potato dextrose agar; ANA, antinuclear antibody; BCG, bacilli Calmette-Guerin; SS, Sjogren's syndrome; SSA, Sjogren's syndrome A; IFN, interferon; DHEA, dehydroepiandrosterone.

\section{Ethical Approval}

The patient's informed consent for publication of the case details including the images was obtained and the study was approved by Ethics Committee of the Second Hospital of Jilin University.

\section{Author Contributions}

All authors made a significant contribution to the work reported, whether that is in the conception, study design, execution, acquisition of data, analysis and interpretation, or in all these areas; took part in drafting, revising or critically reviewing the article; gave final approval of the version to be published; have agreed on the journal to which the article has been submitted; and agree to be accountable for all aspects of the work.

\section{Funding}

There is no funding to report.

\section{Disclosure}

The authors declare no conflicts of interest in this work.

\section{References}

1. Wallace RJ Jr, Brown BA, Onyi GO, et al. Skin, soft tissue, and bone infections due to Mycobacterium chelonae chelonae: importance of prior corticosteroid therapy, frequency of disseminated infections, and resistance to oral antimicrobials other than clarithromycin. $J$ Infect Dis. 1992;166:405-412. doi:10.1093/infdis/166.2.405

2. Lin SS, Lee CC, Jang TN. Soft tissue infection caused by rapid growing mycobacterium following medical procedures: two case reports and literature review. Ann Dermatol. 2014;26:236-240. doi:10.5021/ad.2014.26.2.236 
3. Griffith DE, Aksamit T, Brown-Elliott BA, et al. An official ATS/ IDSA statement: diagnosis, treatment, and prevention of nontuberculous mycobacterial diseases. Am $J$ Respir Crit Care Med. 2007;175:367-416. doi:10.1164/rccm.200604-571ST

4. Bhattacharya J, Mohandas S, Goldman DL, et al. Nontuberculous mycobacterial infections in children. Pediatr Rev. 2019;40:179-190. doi:10.1542/pir.2018-0131

5. Brown-Elliott BA, Philley JV, Schlossberg D. Rapidly growing mycobacteria. Microbiol Spectr. 2017;5(1). doi:10.1128/microbiolspec.TNMI7-0027-2016

6. Lee WJ, Kang SM, Sung H, et al. Non-tuberculous mycobacterial infections of the skin: a retrospective study of 29 cases. J Dermatol. 2010;37:965-972. doi:10.1111/j.1346-8138.2010.00960.x

7. Brito-Zeron P, Retamozo S, Ramos-Casals M. Phenotyping Sjogren's syndrome: towards a personalised management of the disease. Rheumatol Int. 2018;36:S198-S209.

8. Price EJ, Baer AN. How to treat Sjogren's syndrome. Rheumatology. 2021;60:2574-2587. doi:10.1093/rheumatology/key363
9. Dow CT, Chan ED. What is the evidence that mycobacteria are associated with the pathogenesis of Sjogren's syndrome? J Transl Autoimmun. 2021;4:100085. doi:10.1016/j.jtauto.2021.100085

10. Chao W-C, Lin C-H, Liao T-L, et al. Association between a history of mycobacterial infection and the risk of newly diagnosed Sjogren's syndrome: a nationwide, population-based case-control study. PLoS One. 2017;12:e176549. doi:10.1371/journal.pone.0176549

11. Nezos A, Gravani F, Tassidou A, et al. Type I and II interferon signatures in Sjogren's syndrome pathogenesis: contributions in distinct clinical phenotypes and Sjogren's related lymphomagenesis. J Autoimmun. 2015;63:47-58. doi:10.1016/j.jaut.2015.07.002

12. Rayamajhi M, Humann J. Induction of IFN alpha beta enables Listeria monocytogenes to suppress macrophage activation by IFN gamma. Virulence. 2010;1(5):418-422. doi:10.4161/viru.1.5.12787
Infection and Drug Resistance

\section{Publish your work in this journal}

Infection and Drug Resistance is an international, peer-reviewed openaccess journal that focuses on the optimal treatment of infection (bacterial, fungal and viral) and the development and institution of preventive strategies to minimize the development and spread of resistance. The journal is specifically concerned with the epidemiology of
Dovepress

antibiotic resistance and the mechanisms of resistance development and diffusion in both hospitals and the community. The manuscript management system is completely online and includes a very quick and fair peerreview system, which is all easy to use. Visit http://www.dovepress.com/ testimonials.php to read real quotes from published authors. 